\title{
A Planar Capacitive Precision Gauge for Liquid-Level and Leakage Detection
}

\author{
Ferry N. Toth, Gerard C. M. Meijer, Member, IEEE, and Matthijs van der Lee
}

\begin{abstract}
A new capacitive precision liquid-level gauge has been developed. The sensor consists of a low-cost planar electrode structure, a capacitance-controlled oscillator and a microcontroller. The device is able to measure absolute levels of conducting and nonconducting liquids with a $1 \mathrm{~mm}$ uncertainty over a $4 \mathrm{~m}$ range. The system has a high resolution of $0.1 \mathrm{~mm}$ at short measuring times of only $0.2 \mathrm{~s}$. Slow level movements of $0.02 \mathrm{~mm} / \mathrm{h}$ can be detected within $18 \mathrm{~min}$.
\end{abstract}

\section{INTRODUCTION}

$\mathbf{M}$ ANY DIFFERENT types of liquid-level sensors are used throughout industry. The sensor presented here attempts to improve a type of sensor used in storage tanks at fuel service stations [1]. These tanks usually contain nonconductive liquids like leaded or unleaded gasoline or diesel fuel. However sometimes conductive liquids like water need to be measured, for instance for calibration purposes. Liquid-level gauges can also be used to detect a leakage in the tank. For this application a high resolution is required.

In this paper a capacitive sensor with a high resolution is presented, that uses a new low-cost electrode structure. This sensor is able to measure simultaneously the levels of both conducting and nonconducting liquids with an equal accuracy.

\section{Physical Aspects}

The electrode structure spans the whole measuring range and has a maximum length of nearly $4 \mathrm{~m}$. It consist of a long electrode $\left(E_{0}\right)$ and one that is divided into insulated segments $\left(E_{1} \cdots E_{n}\right)$. The relative position of the electrodes is fixed by the mechanical construction, while the absolute position requires a one-time calibration. All capacitances are connected to either the low-impedance voltage source, the low-impedance measurement-system input or to ground (Fig. 1).

The level of nonconducting liquids can be calculated by finding the interface segment $i$, which has a value between $C_{i-1}$, the capacitance in the liquid and $C_{i+1}$, the capacitance in air. Then the capacitance of the interface segment $C_{i}$ can

Manuscript received June 20, 1996; revised October 1, 1996. This work was supported by the Dutch Foundation for Technical Sciences (STW) and ENRAF BV.

F. N. Toth and M. van der Lee are with ENRAF BV, Delft Instruments Group, 2624 BD Delft, The Netherlands.

G. C. M. Meijer is with the Faculty of Electrical Engineering, Delft University of Technology, 2628 CD Delft, The Netherlands.

Publisher Item Identifier S 0018-9456(97)01583-0.

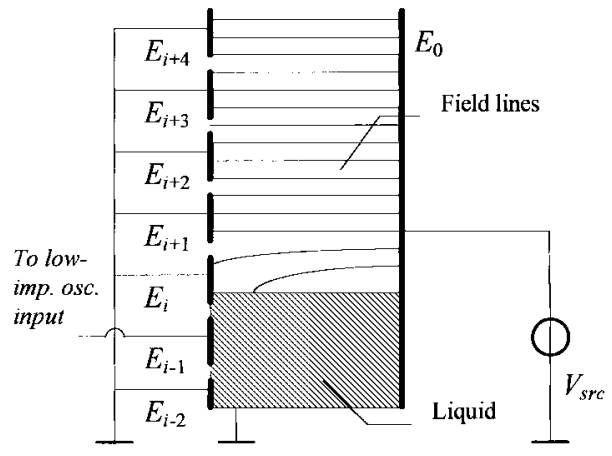

Fig. 1. Conductive liquid connected to ground.

be interpolated to find the interface position $p$ accurately

$$
\begin{aligned}
p & =l \cdot\left(\frac{\frac{C_{i}}{C_{\mathrm{ref}}}-\frac{C_{i+1}}{C_{\mathrm{rcf}}}}{\frac{C_{i-1}}{C_{\mathrm{ref}}}-\frac{C_{i+1}}{C_{\mathrm{ref}}}}+i\right) \\
& =l \cdot\left(\frac{C_{i}-C_{i+1}}{C_{i-1}-C_{i+1}}+i\right)
\end{aligned}
$$

where $l$ is the length of the electrode segments.

To measure conducting liquids such as water, a special provision has to be made: To prevent short-circuiting of the input of the measurement system the electrodes need to be covered with an insulating sleeve. This material also protects the electrodes against the possibly aggressive environment. In the proposed setup (Fig. 1) some electric-field bending around the interface will occur.

Assuming a parallel plate electrode structure, covered with an infinitely thin insulator, the conducting liquid can be regarded as a shield (Fig. 1) that is connected to ground. The capacitance between a single electrode segment $E_{i}$ and the opposite electrode $E_{0}$ can be calculated as a function of the interface level. The nonlinearity in the characteristic is caused by field-bending effects.

The parallel plate electrode structure has the advantage of a simple physical structure that can easily be modeled. However, the costs of constructing such a structure with a length up to $4 \mathrm{~m}$ are considerable.

To reduce costs, the transmitting electrode segments and the receiving electrode can be integrated on the same substrate. Shi et al. [2] presented a structure where electrode $E_{0}$ is removed and the capacitances are measured between the successive electrodes $E_{i}$ and $E_{i+1}$. This method leads to a rather complex model to retrieve the level from the measured capacitances. An alternative approach is to integrate electrode $E_{0}$ also 


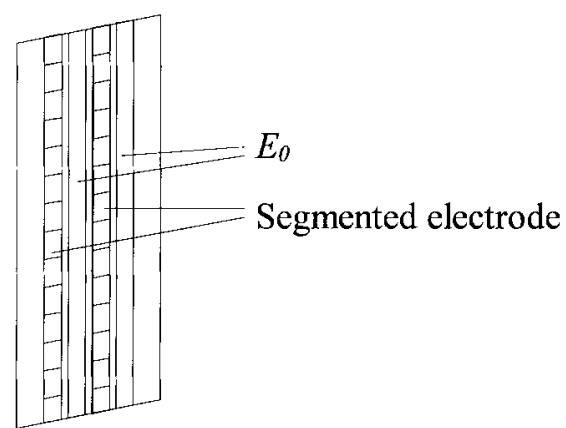

Fig. 2. Novel planar electrode structure.

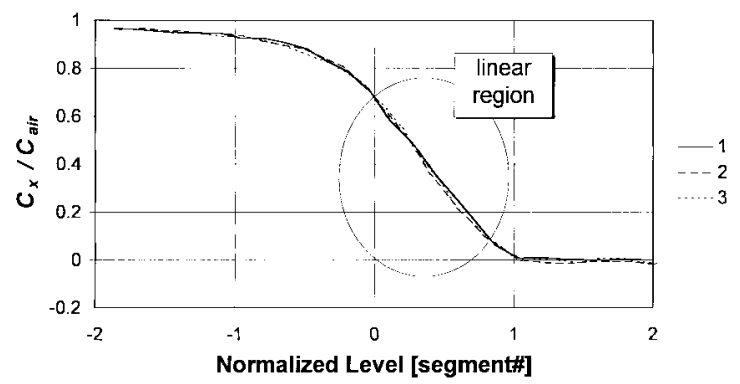

Fig. 3. Measured capacitance ratios versus interface level for conductive liquids.

on the same substrate. Such a structure has been suggested previously by Heerens et al. [3]. The prototype presented there was a circular version with the electrodes on the outside of a glass tube, which is also not very suitable for low-cost manufacturing. However, by projecting this circular structure on a plane, the novel planar electrode structure has been obtained (Fig. 2).

To reduce the effect of nonidealities near the electrode surface, a thin guard has been placed between them. The resulting capacitance per segment length in air is only $2 \mathrm{fF} / \mathrm{mm}$. Therefore the whole structure is duplicated which results in 3 capacitances that are connected in parallel. The resulting structure has electrode segments that have a capacitance per segment of about $6 \mathrm{fF} / \mathrm{mm}$ in air and $12 \mathrm{fF} / \mathrm{mm}$ in unleaded fuel.

Similar field bending effects occur as with the biplanar structure. The effect on the capacitance has been measured for conductive (Fig. 3) and nonconductive liquids (Fig. 4).

For conductive liquids (tap water) we can see that when the electrode is completely covered with liquid (Level $>1$ ) that the capacitance is very near to zero. When the capacitance is completely uncovered (Level $<0$ ) the relative capacitance approaches 1 (the capacitance of air) the further the interface is away. Between 0 and 1 the curve is S-shaped, with a linear region in the middle. It can be shown that by connecting consecutive segments in parallel, a segment with a effective length equal to a multiple of the segment length is created. This way the linear region increases with a multiple of the segment length and the nonlinearity can be reduced to an insignificant level. The asymmetry of the curve in Fig. 3 merely causes an offset that can be calibrated in the factory.

For nonconductive liquids a similar curve can be obtained. In Fig. 4 the results are shown for unleaded car fuel. Some

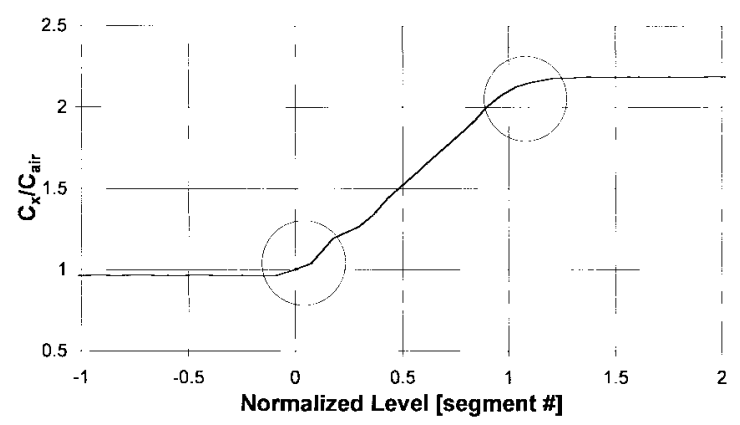

Fig. 4. Measured capacitance ratio's versus interface level for nonconductive liquids.

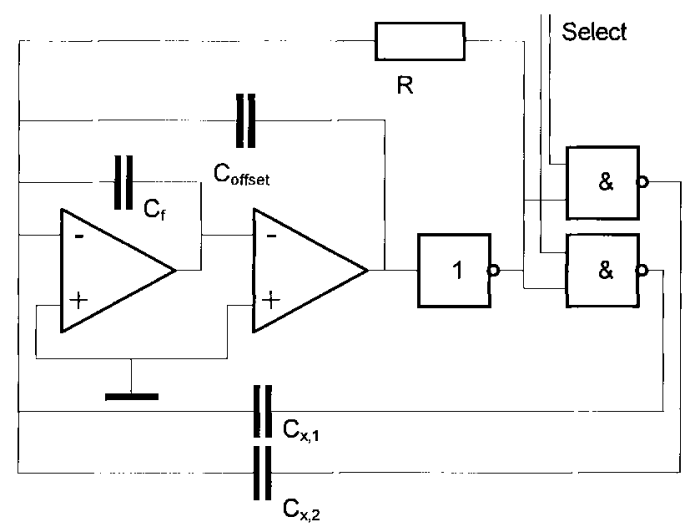

Fig. 5. The capacitance controlled oscillator.

field bending also occurs in this case due to the isolating sleeve, causing the rounded corners of the curve. The nonlinearity can be reduced in a similar way as for conductive liquids. In this case however, the offset is negligible due to the symmetry of the curve.

\section{Capacitance Measurement}

The most important tasks for the capacitance measurement system are to select the correct capacitance, to eliminate the effect of the large parasitic capacitances caused by the long wires and to convert the capacitance to a form that is compatible with a microcontroller input.

This has been achieved by connecting the capacitances to a capacitance-controlled relaxation oscillator (Fig. 5) [4]. In this oscillator there is a linear relationship between the capacitance and the period

$$
T_{x, i}=4 R\left(C_{\text {offset }}+C_{x, i}\right)
$$

Using a multiplexer that is controlled by a microcontroller as has been suggested by Toth and Meijer [5], [6], the capacitances $C_{\text {offset }}, C_{\text {offset }}+C_{x, 1}$ and $C_{\text {offset }}+C_{x, 2}$ can be selected independently. This results in the periods $T_{\text {offset, }} T_{x, 1}$ and $T_{x, 2}$, respectively. The microcontroller measures the period of the oscillator and calculates the capacitances. By applying an autocalibration technique called the three-signal approach,

$$
\frac{C_{x, 1}}{C_{x, 2}}=\frac{T_{x, 1}+T_{\text {offset }}}{T_{x, 2}+T_{\text {offset }}}
$$




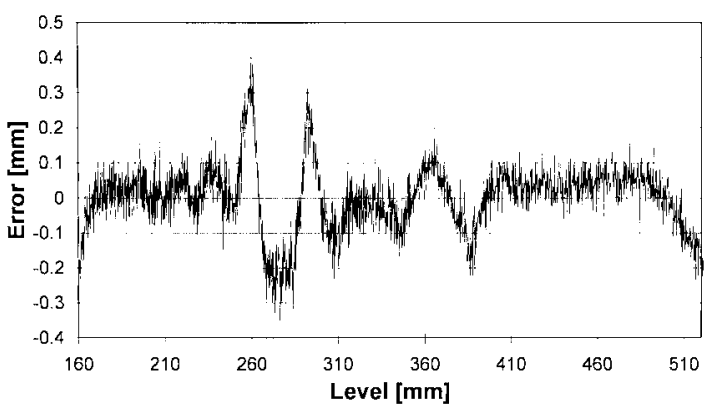

Fig. 6. The total error for Euro Super over a measurement range of $300 \mathrm{~mm}$

all additive and multiplicative errors are reduced to insignificant levels.

This system measures capacitances of $100 \mathrm{fF}$ with an accuracy of $50 \mathrm{aF}$ with respect to the reference capacitor within $100 \mathrm{~ms}$. The influence of grounded parasitic capacitances up to $800 \mathrm{pF}$ is reduced to an insignificant level.

The microcontroller has been programmed to either measure selected capacitances or to search for and track the interface segment $i$ and calculate the position $p$. A PC is used to query the calculated position.

\section{EXPERIMENTAL RESULTS}

A complete sensor has been fabricated consisting of two multilayer printed circuits boards (PCB) with 48 electrodes of $8 \mathrm{~mm}$ each and $1 \mathrm{PCB}$ with the capacitance measurement circuit. The PCB's are glued to a glass filled plastic carrier to accurately fix the position of the electrodes. The switches that are required to select the appropriate capacitor are mounted on the back side of the PCB's. One of the layers is connected to ground, shielding the electrodes from the switches.

A fully automated measurement setup has been constructed, to test several different types of car fuels, such as unleaded super and diesel but also tap water. The setup consists of a vertical tank with a height of $1.2 \mathrm{~m}$ containing $0.01 \mathrm{~m}^{3}$ liquid. At the bottom of the tank is a valve that is connected to the reservoir. A computer controlled pump pumps the liquid back from the reservoir to the tank. When the valve is opened slightly, the liquid level decreases from about $500 \mathrm{~mm}$ to $160 \mathrm{~mm}$ in about $2 \mathrm{~h}$. The PC is used to store the measured level every $2 \mathrm{~s}$.

By fitting a second order polynomial, the error can be determined as a function of the level (Fig. 6). Due to the fitting process, the calculated error excludes the absolute position offset, the scale error and the second order term (which can be assumed to be negligible). The absolute position offset is determined by a one-time factory calibration. The scale error is determined by the positioning of the PCB's and by the thermal expansion coefficient of the carrier. Therefore these errors are only determined by the manufacturing process and the materials used.

With Euro Super, the remaining error is less than $\pm 0.4 \mathrm{~mm}$, while the resolution $(3 \sigma)$ is about $0.1 \mathrm{~mm}$ with a $0.2 \mathrm{~s}$ measuring time. With tap water and diesel similar results have been obtained. It has been found that when this system is used for leak detection in a $10 \mathrm{~m}^{3}$ tank, a $0.02 \mathrm{~mm} / \mathrm{h}$ level change (caused by leak) can be detected in only 18 minutes. For a $10 \mathrm{~m}^{3}$ tank this corresponds to a leak rate of $0.4 \mathrm{l} / \mathrm{h}$.

\section{CONCLUSION}

A novel, capacitive fuel level gauge for fuel storage tanks has been presented. In the gauge a low-cost, planar electrode structure has been applied. The capacitance is converted to a period by a relaxation oscillator which is measured by a microcontroller. By mounting the circuitry on the back side of the electrodes, the capacitances can be measured accurately and at low-costs.

The gauge is suitable for the simultaneous measurement of water and fuel levels, with an accuracy of $0.4 \mathrm{~mm}$ within a $0.2 \mathrm{~s}$ measuring time. The high resolution $(0.1 \mathrm{~mm})$ allows the gauge to be used for leakage detection.

\section{REFERENCES}

[1] "Level gauging system for service stations," User Manual, B. V. EnrafNonius, Delft, The Netherlands, 1983.

[2] T. M. Shi, M. S. Beck, S. M. Huang, R. A. Williams, and C. G. Xie, "Capacitance-based instrumentation for multi-interface level measurement," Meas. Sci. Technol., vol. 2, pp. 923-933, Oct. 1991.

[3] W. C. Heerens, G. Keizer, and F. W. Wedman, "Theory and practice of multi-terminal capacitor bar and tube sensors for chemical and physical process applications," in Proc. Sensor'83, Basel, Switzerland, May 1983, vol. 5 , pp. 27-40, 17-19.

[4] J. van Drecht, "Relaxatie oscillator," Patent 91.01076, The Netherlands, 1991.

[5] F. N. Toth and G. C. M. Meijer, "A low-cost, smart capacitive position sensor," IEEE Trans. Instrum. Meas., vol. 41, pp. 1041-1044, Dec. 1992

[6] F. N. Toth, H. M. M. Kerkvliet, and G. C. M. Meijer, "A very accurate measurement system for multi-electrode $\mathrm{pF}$-range capacitors," IEEE Trans. Instrum. Meas., vol. 45, pp. 531-535, Apr. 1996.

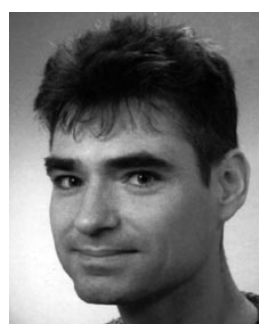

Ferry N. Toth was born in Sydney, Australia, on August 4, 1966. He received the ingenieurs (M.S.) and Ph.D. degrees in electrical engineering from the Delft University of Technology, Delft, The Netherlands, in 1990 and 1997, respectively.

From 1990 to 1991, he was with Delft Instruments Tang Gauging BV, Delft, formerly known as Enraf Nonius, where he was engaged in the development of a capacitive level gauge. After this he was with the Delft University of Technology, where he was involved in scientific research on intelligent capacitive sensors. He is currently with ENRAF BV, Delft Instruments Group, Delft, and is involved in the development of a new generation of intelligent capacitive level gauges.

Gerard C. M. Meijer, (M'94) for a photograph and biography, see this issue, p. 639.

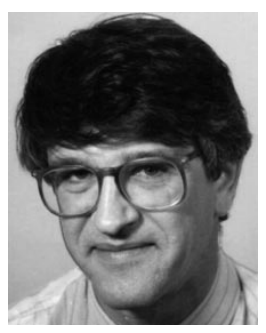

Matthijs van der Lee was born in 1948. He received the M.S. degree in electrical engineering from the Delft University of Technology, Delft, The Netherlands, in 1976.

He was first involved in designing control circuits for power electronic circuits at Holec and is presently with the instruments manufacturing company ENRAF BV, Delft Instruments Group, Delft, The Netherlands, as Head of the Development Department. His special interests are the design of microwave and capacitive level gauges. 